\title{
SIMULATION OF DENSITY CHANNEL GUIDING IN CAPILLARY DISCHARGE EXPERIMENTS AND LASER WAKEFIELD ACCELERATORS
}

\author{
$\underline{\text { R. F. Hubbard }},{ }^{\text {a\# }}$ C. Moore, ${ }^{a}$ P. Sprangle, ${ }^{a}$ A. Ting, ${ }^{a}$ NRL, D. Kaganovich, ${ }^{b}$ A. Zigler ${ }^{b}$ and B. Hafizi ${ }^{\mathrm{c}}$
}

\begin{abstract}
Laser-driven accelerators require guiding of the laser pulse over many Rayleigh lengths. Plasma channels such as those produced by a capillary discharge have been shown experimentally to be capable of guiding intense laser pulses over distances as long as $6 \mathrm{~cm}$. Simulations of laser propagation in these channels show that the laser pulse radius undergoes oscillations about the matched radius at the expected frequency. The pulse may be distorted by several effects, including laser-generated ionization. Experiments to date have been at relatively high densities $\left(10^{18}-10^{19} \mathrm{~cm}^{-3}\right)$. For standard laser wakefield accelerator applications, the channel density is likely to be substantially lower. As expected, simulations in this lower density regime show lower accelerating gradients, larger laser spot sizes, and higher wakefield phase velocities and dephasing-limited energy gain.
\end{abstract}

\section{INTRODUCTION}

The laser wakefield accelerator (LWFA) [1,2] has emerged as a leading candidate among various advanced accelerator concepts. However, the single-stage energy gain will be severely limited by diffraction of the laser pulse unless the beam is optically guided. Preformed plasma channels offer a promising method for providing such guiding. Channels produced by an axicon-focused laser [3] or capillary discharge [4-7] have successfully guided laser pulses over distances of several $\mathrm{cm}$.

Several LWFA experiments $[8,9]$ have produced very large accelerating gradients $(>10 \mathrm{GV} / \mathrm{m})$ and high energies $(\sim 100 \mathrm{MeV})$, but these experiments operate in the highly-unstable self-modulated regime and produce poor beam quality. Experiments in the standard LWFA regime have to date produced moderate energy gain [10].

This paper describes simulations of laser pulses in plasma channels similar to those used in recent capillary discharge guiding experiments. The analysis is extended the lower plasma density regime which will be used in future channel-guided LWFA experiments.

\footnotetext{
"Work supported by the Dept. of Energy, the Office of Naval Research, and the US-Israeli Binational Science Foundation.

a Plasma Physics Division, Naval Research Laboratory, Washington, DC

${ }^{\mathrm{b}}$ Racah Institute of Physics, Hebrew University, Jerusalem, Israel

'Icarus, Inc., Bethesda, MD

\#Email: hubb@ppdu.nrl.navy.mil
}

\section{LASER PROPAGATION IN DENSITY CHANNELS}

\subsection{Theory for an Ideal Density Channel}

Refractive guiding of a laser pulse can occur if the index of refraction peaks on-axis [2-7,11]. A plasma channel with an on-axis density minimum produces the desired effect. For an ideal channel, the plasma density is assumed to be parabolic, with $n=n_{0}+\Delta n\left(r / r_{c h}\right)^{2}$, where $n_{0}$ is the on-axis density, $r$ is the distance from the axis, $\Delta n$ is the channel depth, and $r_{c h}$ is the channel radius. The laser pulse is assumed to have a Gaussian radial profile, with normalized vector potential $a=a_{0} \exp \left(-r^{2} / r_{L}^{2}\right)$, where $r_{L}$ is the laser spot size.

The assumptions above lead to an envelope equation for $r_{L}(z)$ in which the spot size oscillates about the equilibrium or matched radius $r_{M}$ given by

$$
r_{M}=\left(r_{c h}^{2} / \pi r_{e} \Delta n\right\}^{1 / 4},
$$

where $r_{e}$ is the classical electron radius. The oscillations have a period given by $\lambda_{e}=\pi Z_{M}$, where $Z_{M}=\left(\pi r_{M}\right)^{2} / \lambda$ is the Rayleigh length for the matched spot size $r_{M}$.

\subsection{Laser Guiding Experiments with Capillary Discharges}

A capillary discharge provides a simple, controllable method for generating a narrow plasma column suitable for laser guiding [4-7]. The device consists of a thin cylinder of insulating material with high voltage electrodes at the ends. The plasma is generated from material from the inner insulating wall of the capillary. Double capillary designs [12] employ a short higher voltage trigger section preceding the main capillary. Hydrodynamic simulations [12] and Stark broadening measurements [5] indicate a period of time during which the plasma has an off-axis maximum in the density at a distance $r_{c h}$ which is typically $50-70 \%$ of the wall radius.

Initial experiments $[4,5]$ utilized a linearly-polarized Ti-sapphire laser with pulse length $\tau_{L}=100 \mathrm{fs}$, wavelength $\lambda=0.8 \mu \mathrm{m}$, and peak power $P_{o}$ up to 0.1 TW. The pulses were focused onto the entrance of the capillary with a focused spot size of $15 \mu \mathrm{m}$. Experiments with $1 \mathrm{~cm}$ long cylindrical capillaries [4] demonstrated transport efficiencies of up to $85 \%$ in both straight and curved configurations. When the discharge was not 
triggered, transport efficiencies dropped dramatically, and the transported laser spot appeared to fill the capillary. Laser guiding experiments were also performed in 2, 3 and $6.6 \mathrm{~cm}$ long capillaries [5]. In the latter case, the pulse radius at capillary exit was $<25 \mu \mathrm{m}$ and did not vary greatly with variations of the discharge parameters. The transport distance was $\sim 30 Z_{M}$. Light transmission was $\sim 10 \%$ in comparison to the $\sim 1 \%$ without the discharge. Collisional absorption is likely to be the dominant loss mechanism, although ionizationinduced losses cannot be discounted [5].

Accelerator applications require substantially higher intensities than are produced by this laser. To investigate the high intensity regime, capillary discharge experiments have recently been carried out using the $T^{3}$ laser at the Naval Research Laboratory [6,7]. The laser has a $1.06 \mu \mathrm{m}$ wavelength, a $400 \mathrm{fs}$ pulse length, and peak power exceeding $2 \mathrm{TW}$. A $2 \mathrm{~cm}$ long double capillary was used. The focused intensities in these experiments exceeded $10^{17} \mathrm{~W} / \mathrm{cm}^{2}$. Transport efficiencies were generally higher than at lower intensities

\subsection{Laser Guiding Simulations}

Simulations of laser propagation in plasma channels have been carried out using the LEM 2-D, axisymmetric code developed by Krall, et al. [13]. Nonlinear wave equations are solved for the normalized potentials, and the plasma response is treated with a cold fluid model. The evolution of the pulse is followed in a speed-of-light system with coordinates $r, \zeta=c t-z$, and $\tau=t$.

Figure 1 plots the spot size $r_{L}(z)$ for three simulations in a $2 \mathrm{~cm}$ long channel with $r_{c h}=100 \mu \mathrm{m}$, similar to that used in the guiding experiments described above [5]. The spot size is calculated at a reference point near the center of the $100 \mathrm{fs}$ long, $0.8 \mu \mathrm{m}$ wavelength pulse. The entrance to the channel is near the initial focus at $z=0.3$ $\mathrm{cm}$, and the pulse exits the channel into vacuum at $z=$ $2.3 \mathrm{~cm}$. The solid curve has $n_{0}=5 \times 10^{18} \mathrm{~cm}^{-3}$, and $\Delta n=$ $3 \times 10^{18} \mathrm{~cm}^{-3}$ and is nearly identical to the dashed curve, which raises $n_{0}$ to $1.5 \times 10^{19} \mathrm{~cm}^{-3}$. However, the dotted curve, which has a small reduction in $\Delta n\left(2.8 \times 10^{18} \mathrm{~cm}^{-3}\right)$, produces a small but noticeable shift in the spot size oscillation wavelength $\lambda_{e}$, leading to a significant shift in the spot size trajectory after it exits the channel. This is consistent with the predictions of Section 2.1, that $r_{M}$ and $\lambda_{e}$ are independent of $n_{0}$ and scale only with $\Delta n$. Experimental measurements of post-capillary expansion of the beam show shot-to-shot variations which can be attributed to small changes in $\Delta n$.

The pulse may be distorted by a number of effects which are not contained in the simple envelope models. For example, the intense laser pulse may ionize the carbon originating from the polyethylene capillary wall to higher charge states. This will tend to increase the plasma density near the axis, which inhibits the guiding effects and may cause the pulse profile to hollow [4].
Finite pulse length effects [14] may also distort the pulse, causing growth and damping of envelope oscillations.

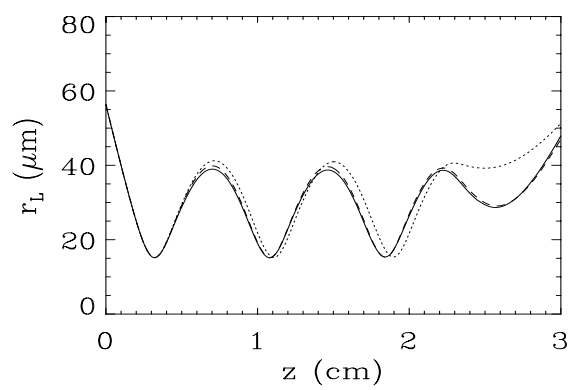

Fig. 1. Simulation results for laser guiding and postcapillary expansion for three cases with different values of $n_{0}$ and $\Delta n$. Parameters are described in the text.

\section{CHANNEL-GUIDED LASER WAKEFIELD ACCELERATORS}

\subsection{Scaling for LWFA Performance}

The channel guiding method described above may be adapted to laser wakefield acceleration. However, to be in the optimal regime for a standard LWFA, the resonance ratio $\alpha_{r}=c \tau_{L} / \lambda_{p}$ should be $\sim 0.5[1,2]$. Here, the nominal plasma wavelength $\lambda_{p}=2 \pi c / \omega_{p 0}$, and $\omega_{p 0}=$ $\left(4 \pi n_{0} e^{2} / m\right)^{1 / 2}$ is the on-axis plasma frequency. If $\alpha_{r}$ and $\tau_{L}$ are specified, the on-axis density $n_{0}=\left(\pi m / e^{2}\right)\left(\alpha_{r} / \tau_{L}\right)^{2}$. For $\tau_{L}>100 \mathrm{fsec}$, and $\alpha_{r}=0.5, n_{0}<3.5 \times 10^{17} \mathrm{~cm}^{-3}$, which is much lower than $n_{0}$ values in current experiments [3-7].

Analytical models for predicting LWFA performance are usually based on such physics parameters as $a_{0}, \lambda$, and $\lambda_{p}$. The primary performance quantities are the peak accelerating electric field $E_{m}$, the dephasing distance $L_{d}$, and the dephasing-limited energy gain $W_{d}=m c^{2} \gamma_{d}$. If the pulse length is optimized, the pulse is linearly-polarized, and $a_{0} \ll<1$, the usual scalings give $E_{m}=\left(a_{0}^{2} / 2\right) E_{0}, L_{d}=$ $\lambda_{p}{ }^{3} / 2 \lambda^{2}$, and $\gamma_{d}=2\left(E_{m} / E_{0}\right)\left(\lambda_{p} / \lambda\right)^{2}$. Here $E_{0}=m c \omega_{p o} / e$ is the 1-D cold fluid wavebreaking field, and the dephasing limits are based on the distance over which a particle moving at $c$ slips by an $\lambda_{p} / 4$ in the wakefield.

Since $\alpha_{r}$ and $\tau_{\mathrm{L}}$ are strongly linked, one may express performance quantities in terms of the following set of laser parameters $\left(\lambda, P_{0}, \tau_{\mathrm{L}}\right)$ and channel parameters $\left(\alpha_{r}\right.$, $r_{c h}$, and normalized channel depth $\left.\Delta n / n_{0}\right)$. The spot size in Eq. (1) is given by $r_{M}{ }^{2}=(c / \pi)\left(r_{c h} \tau_{L}\right) / \alpha_{r}\left(\Delta n / n_{0}\right)^{1 / 2}$. Thus, longer pulse length lasers tend to have larger spot sizes and thus lower intensities and accelerating fields. If the scaling of $E_{m}$ with pulse length is assumed to be $\sim \sin \left(\pi \alpha_{r}\right)$, then the peak accelerating electric field is

$$
E_{m}=\left(\frac{4 e}{m c^{5}}\right) \frac{\alpha_{E} \alpha_{r}^{2} \lambda^{2} P_{0}\left(\Delta n / n_{0}\right)^{1 / 2} \sin \pi \alpha_{r}}{r_{c h} \tau_{L}^{2}}
$$

and the corresponding dephasing-limited energy gain is 


$$
\gamma_{d}=\left(\frac{4 e^{2}}{\pi m^{2} c^{4}}\right) \frac{\alpha_{E} P_{0} \tau_{L}\left(\Delta n / n_{0}\right)^{1 / 2} \sin \pi \alpha_{r}}{r_{c h} \alpha_{r}} .
$$

The scale factor $\alpha_{E}$ is $\sim 0.8[1,2]$. These simple scalings may be modified for high laser intensity $\left(a_{0} \sim 1\right)$ and finite spot size.

\subsection{LWFA Simulations}

The simulation code may be run in the lower density regime and high laser powers appropriate for a LWFA. Fig. 2 gives a surface plot of the axial electric field $E_{z}(r, \zeta)$ for a prototype channel-guided LWFA with $\lambda=0.8 \mu \mathrm{m}, \tau_{L}=100 \mathrm{fs}, P_{0}=25 \mathrm{TW}, n_{0}=2 \times 10^{17} \mathrm{~cm}^{-3}$, $\Delta n / n_{0}=5$, and $r_{c h}=100 \mu \mathrm{m}$. The peak field of $\sim 10$ $\mathrm{GV} / \mathrm{m}$ occurs at $\zeta=-80 \mu \mathrm{m}$, and the simulation slippage rate gives an estimated dephasing length $L_{d s}=20.9 \mathrm{~cm}$. The dephasing-limited energy gain for the simulation is $W_{d s}=(2 / \pi) E_{m s} L_{d s}=1.36 \mathrm{GeV}$. This agrees well with the analytical estimate from the previous section when spot size and nonlinear corrections are included. Current LWFA and channel guiding experiments have taken place at much high plasma densities, where $L_{s} \sim 0.1 \mathrm{~cm}$, $E_{m}$ is substantially higher, but $W_{d}$ is only $\sim 100 \mathrm{MeV}$.

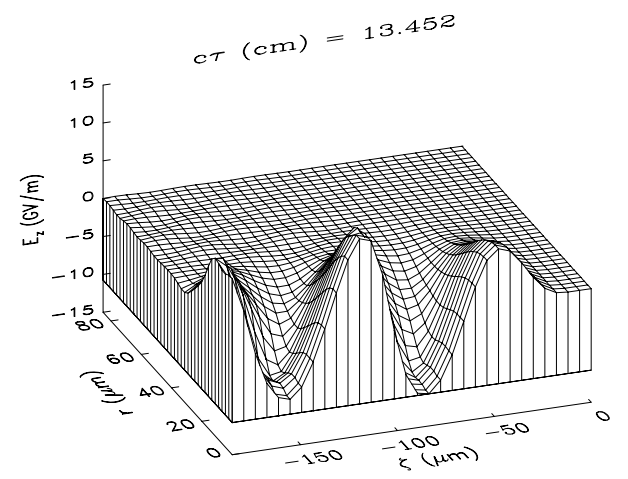

Fig. 2. Axial electric field $E_{z}(r, \zeta)$ at $z=13.5 \mathrm{~cm}$ for the LWFA simulation described above.

Longer pulses require still lower plasma densities but may produce much larger values for $L_{d}$ and $W_{d}$. Simulations with 400 fs long pulses confirm this, but the resulting accelerating gradients are in the $1 \mathrm{GV} / \mathrm{m}$ range. Thus, the channels required to take advantage of the higher dephasing limits are probably too long to be practical.

If a long pulse laser is injected into a higher density channel, the pulse will undergo self modulation (SM) as in the SM-LWFA experiments [8,9]. The channel allows the beam to be guided at powers well below the critical power $P_{c}$ for relativistic self-focusing, and should substantially reduce the highly irregular behavior seen in those experiments. Fig. 3 shows a plot of $E_{z}(r, \zeta)$ at $z=$ $3.6 \mathrm{~cm}$ for a prototype channel-guided SM-LWFA with $\lambda=1 \mu \mathrm{m}, \tau_{L}=400 \mathrm{fs}, P_{0}=13 \mathrm{TW}, n_{0}=8 \times 10^{17} \mathrm{~cm}^{-3}$,
$\Delta n / n_{0}=1$, and $r_{c h}=150 \mu \mathrm{m}$. The simulation produces a well-defined wakefield which peaks at $\sim 35 \mathrm{GV} / \mathrm{m}$.

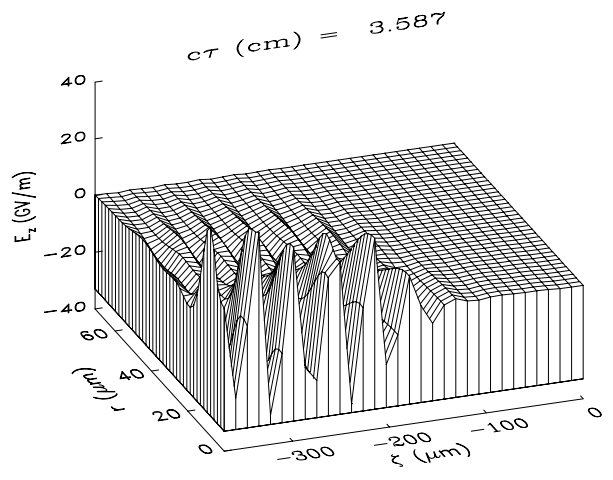

Fig. 3. Axial electric field $E_{z}(r, \zeta)$ at $z=3.6 \mathrm{~cm}$ for the self-modulated LWFA simulation described above

\section{SUMMARY}

Plasma channels such as those produced by a capillary discharge have guided intense laser pulses over distances of several $\mathrm{cm}$. Simulations have been carried out which reproduce these experiments and illustrate higher order effects which may distort the pulse. For a channel-guided LWFA, the plasma density must be substantially lower that in current experiments. The implications may be seen in simple scaling laws for LWFA performance and in simulations of future LWFA experiments. Simulations of a channel-guided, self-modulated LWFA suggest this approach may be promising.

\section{REFERENCES}

[1] P. Sprangle, et al., Appl. Phys. Lett. 53, 2146 (1988).

[2] E. Esarey, et al., IEEE Trans. Plasma Sci. 24, 252 (1996).

[3] C. G. Durfee, III and H. M. Milchberg, Phys. Rev. Lett. 71, 2409 (1993);H. M. Milchberg, et al., Phys. Plasmas 3, 2149 (1996).

[4] Y. Ehrlich, et al., Phys. Rev. Lett. 77, 4186 (1996).

[5] Y. Ehrlich, et al., J. Opt. Soc. Am. B 15, 2416 (1998).

[6] D. Kaganovich, et al., to appear in Phys. Rev. E.

[7] R. F. Hubbard, et al., to appear in Advanced Accelerator Concepts, AIP Conference Proceedings, 1999.

[8] K. Nakajima, et al., Phys. Rev. Lett. 74, 4659 (1995); A. Modena, et al., IEEE Trans. Plasma Sci. PS-24, 289 (1996); D. Umstadter, et al., Science 273, 472 (1996).

[9] C. Moore, et al., Phys. Rev. Lett. 79, 3909 (1997); A. Ting, et al., Phys. Plasmas 4,1889 (1997).

[10] F. Amiranoff, et al., Phys. Rev. Lett. 81, 995 (1998).

[11] P. Sprangle and E. Esarey, Phys. Fluids B 4, 2241 (1992).

[12] D. Kaganovich, et al., Appl. Phys. Lett. 71, 2295 (1997).

[13] J. Krall, et al., Phys. Rev E 48, 2157 (1993).

[14] P.Sprangle, B. Hafizi, and P. Serafim, Phys. Rev. Lett. 82, 1173 (1999); Phys. Rev. E 59, 3614 (1999); E. Esarey and W. P. Leemans, Phys. Rev. E 59, 1082 (1999). 